\title{
The Disparities of Cancer Incidence between Sudanese Men and Women
}

\author{
Mohammed A Abdulrahman ${ }^{1 *}$, Siddik M Shaheen ${ }^{2}, N^{\prime S}$ Sanh MRS N'dri', AtifElagib4, \\ Alfatih AA Osman ${ }^{1}$, Manal A Elimam ${ }^{5}$ and Sulma Ibrahim Mohammed ${ }^{3}$
}

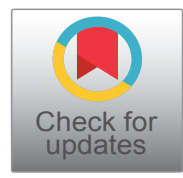

${ }^{1}$ Sudan Atomic Energy Commission (SAEC), Khartoum, Sudan

${ }^{2}$ Faculty of Economic \& Social Studies, University of Khartoum, Khartoum, Sudan

${ }^{3}$ Department of Comparative Pathobiology and Purdue University Center for Cancer Research, Purdue University, West Lafayette IN, USA

${ }^{4}$ National Centre for Research, Khartoum, Sudan

${ }^{5}$ Communicable and Non-communicable diseases (CNCDD) Department, Federal ministry of health (FMOH), Khartoum, Sudan

*Corresponding author: Mohammed Abdulrahman, Sudan Atomic Energy Commission (SAEC), Algamaa Street, PO Box: 3001, Khartoum, Sudan, Tel: +249121821010

\begin{abstract}
Background: Currently, cancer constitutes a public health problem in Sudan. Many factors play a role in increasing the cancer risk in Sudanese people, which include environment, genes, occupation and gender. This study is carried out to investigate the cancer incidence differences between men and women in Sudan.
\end{abstract}

Methods: In this descriptive study, data on 45413patients diagnosed with cancer throughout Sudan over five-year period (2009-2013) were collected and analyzed according to gender. Cancer types $(n=35)$.

Results: The study revealed that in $80 \%$ (28 out of 35 ) of the cancer types examined excluding sex-specific cancers (prostate and breast), male were significantly diagnosed more than females with cancer.

Conclusion: In Sudan, during the period 2009-2013, men were diagnosed more with cancers than women and therefore they may be more susceptible to cancer.

\section{Keywords}

Cancer susceptibility, Gender, Cancer incidence, Sudan, Cancer epidemiology

\section{Introduction}

Cancer was ranked in 2002 as the third leading cause of death among Sudanese peopleafter malaria and pneumonia, accounting for $5 \%$ of all deaths, and as a result constituted a global public health problem [1-3]. Cancer affects both males and females and all agesacross racial and ethnic groups. However, gender disparity in cancer susceptibility exist and this is not restricted to any country, population or specific region [4-6]. The diversities in gender susceptibility was attributed by many to biology, culture, lifestyle, environment, and socioeconomic status between males and females may affect predisposition, development, and cancer clinical presentation between the two groups [7].

A study that analyzed age- and sex-specific cancer incidences conducted by International Agency for Research on Cancer (IARC) and documented in the Cancer Incidence in Five Continents, revealed that cancer incidences among males are higher than incidences among females for 32 out of 35 cancer sites, excluding the sex specific cancers [4]. Additionally, in a study by Cartwright, et al. noticed that the cancer risks for males in various age groups are higher when compared to females [8] and this disparity seems to even exist among children [9].

Despite the knowledge gained from previous studies, less information on the potential sex differences in cancer mortality and cancer survival is available [10]. Gender differences in susceptibility to cancer are very

Citation: Abdulrahman MA, Shaheen SM, N'Sanh MRS N'dri, Elagib A, Osman AAA, et al. (2018) The Disparities of Cancer Incidence between Sudanese Men and Women. Int J Cancer Clin Res 5:101. doi. org/10.23937/2378-3419/1410101

Accepted: November 17, 2018; Published: November 19, 2018

Copyright: (c) 2018 Abdulrahman MA, et al. This is an open-access article distributed under the terms of the Creative Commons Attribution License, which permits unrestricted use, distribution, and reproduction in any medium, provided the original author and source are credited. 


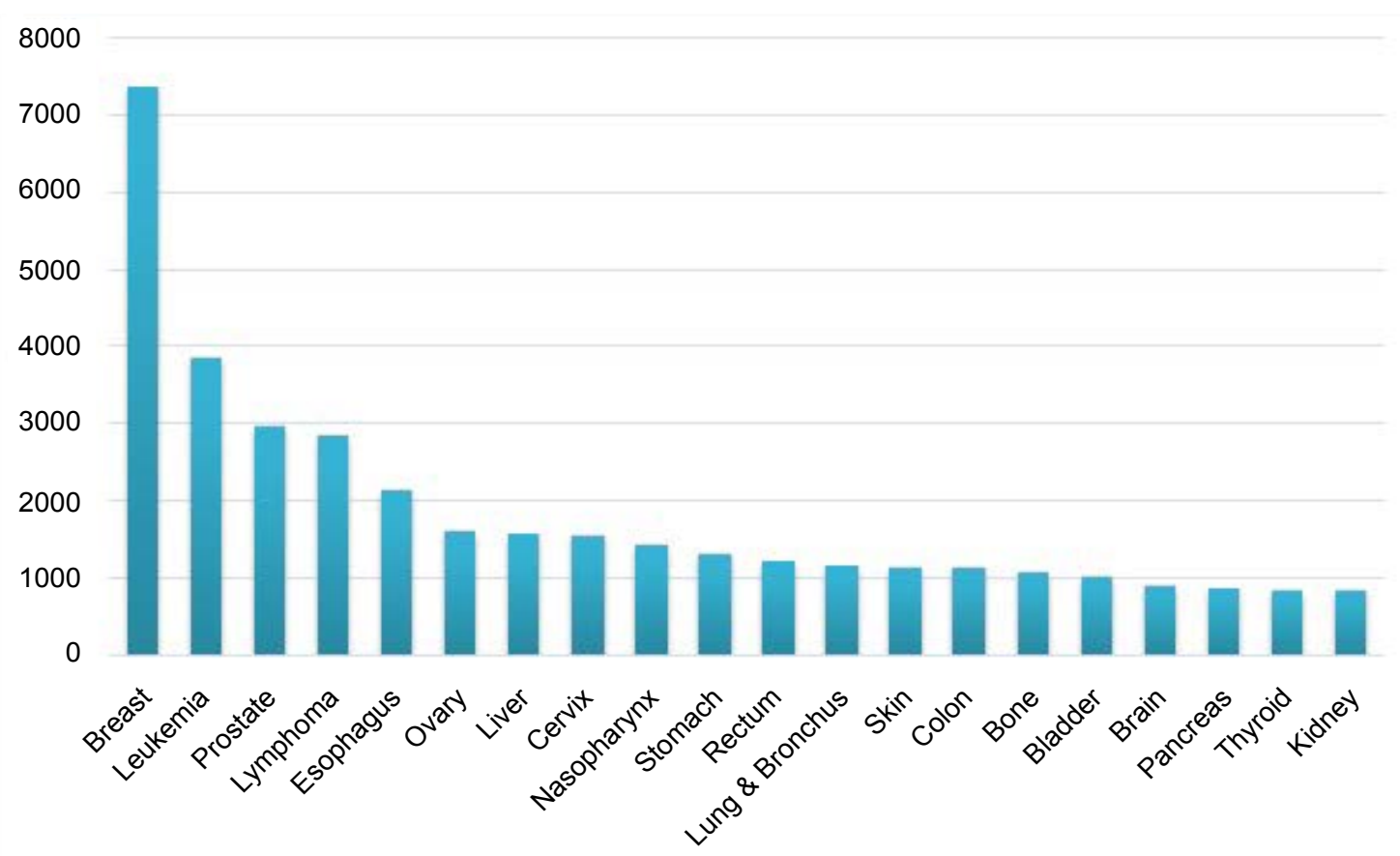

Figure 1: The top 20 cancers among Sudanese males and females from 2009 to 2013.

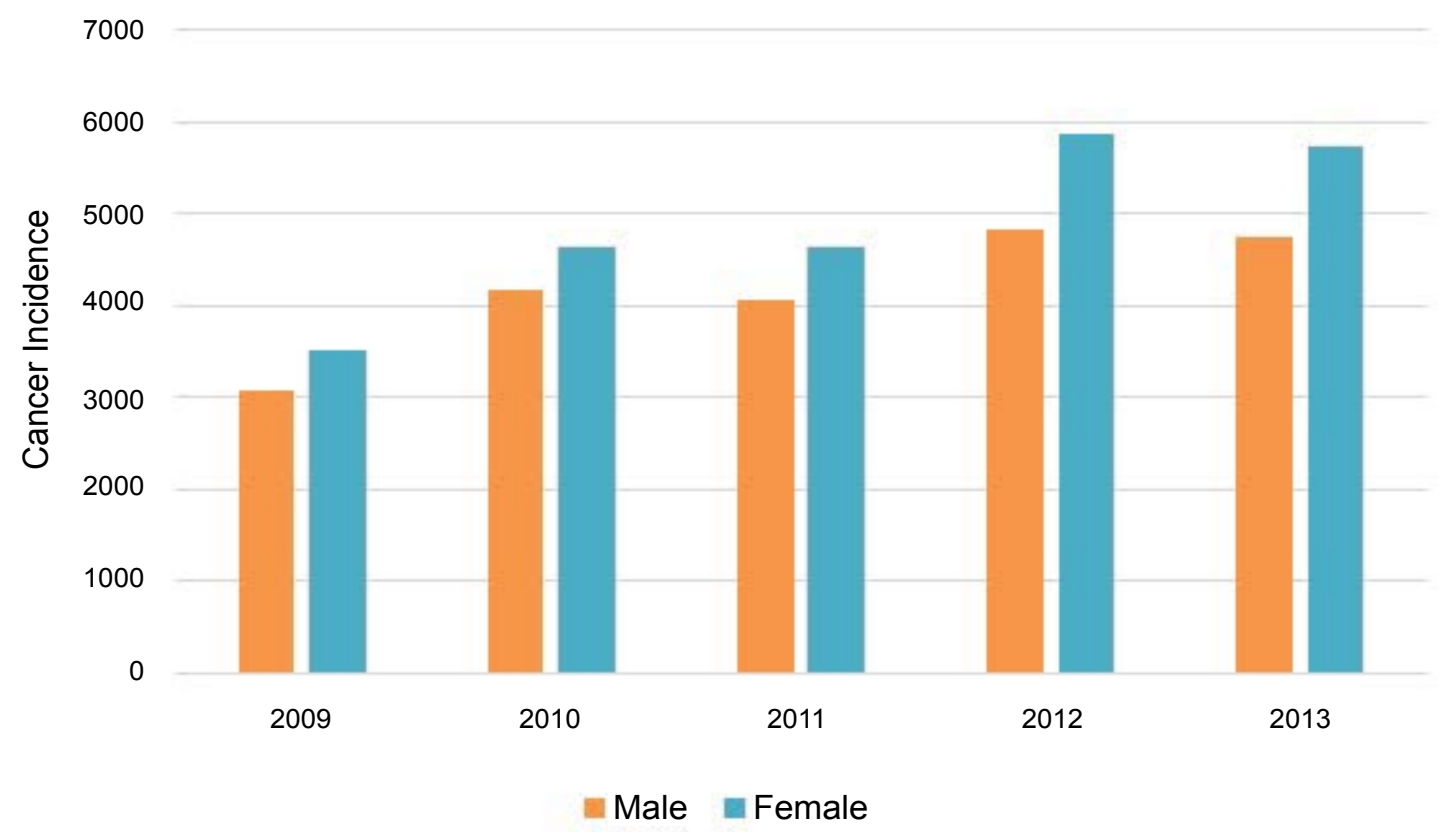

Figure 2: The differences of cancer incidence between males and females during 2009 - 2013.

useful information that can be used to develop a causal hypothesis for the disease, or to define subgroups at highest risk for preventive action [11].

This study has been conducted in Sudan using a population-based data that cover the entire country over a five-year period (2009-2013). The data was then analyzed to determine the effects of gender on cancer incidences in Sudan population.

\section{Material and Methods}

The data have been collected for analysis from the National Cancer Registry of Sudan where 45413 cancer cases were recorded during the years 2009-2013. The method of the study is descriptive in its nature and aims to investigate the disparities of cancer incidence in Sudan among males and females. The Sudan National Cancer Registry abstracts and collects cancer data from all public and private health institutions that provide cancer laboratory diagnosis and treatment, as well as the data from Radiotherapy and Isotope Cancer. The registry uses two methods for data collection, active and passive case finding, covering about 79 private and governmental health facilities and pathological laboratories (18 histopathological laboratories, 33 private, and 28 public hospitals). Sources of data include laboratory reports (histopathology, hematology, and biochemistry), medical record ledgers, and theater's books, patient case notes, and ward admission. Following variables are 
collected for each case: name, age, gender, nationality, tribe, residence, state of residence, date of diagnosis, tumor site, tumor histology, basis of diagnosis, occupation, medical record number, and place of referral. The data used for this study were collected from patients residing in the whole country during the 5-year period 2009-2013.

\section{Results}

Exactly 45413 cancer cases were recorded throughout Sudan over a five-year period (2009-2013). The top 20 common cancers in Sudan are illustrated in Figure 1. Among all registered cancer cases with available information $(\mathrm{N}=45,413)$, breast cancer was the most common type, followed by leukemia and prostate cancer.

Among the 45413 cancer cases studied, 46\% ( $n=$ $20,974)$ were male and $54 \%(n=24,439)$ were female. For all cancer cases including gender specific cancers, females appeared to have the highest number of incidences compared to males (Figure 2). Additionally, the number of new cases per year increased over the 5 years of study.

Then we excluded the gender specific cancers, breast and prostate as they ae the dominant cancer types. As shown in Figure 3 males accounted for more cancer cases than women.
The incidence of the most common sex-specific cancers, breast cancer and prostate cancer, are illustrated through the following Table 1 and Table 2.

The Sudanese males have the higher number of cancer incidences once the gender specific cancers are excluded. After computing the male to female ratio (M: F) for the top 35 cancer incidences, 28 out of 35 cancers showed M: F > 1 . This indicates $80 \%$ cancer susceptibility among males (Figure 4).

Table 1: The frequencies of breast cancer among females during: 2009-2013.

\begin{tabular}{|l|l|l|l|}
\hline Year & Frequency & $\begin{array}{l}\text { Within Total } \\
\text { Cancers (\%) }\end{array}$ & $\begin{array}{l}\text { Within Female } \\
\text { cancers (\%) }\end{array}$ \\
\hline 2009 & 1077 & 2.4 & 30.59 \\
\hline 2010 & 1297 & 2.9 & 27.86 \\
\hline 2011 & 1375 & 3 & 29.59 \\
\hline 2012 & 1841 & 4.1 & 31.36 \\
\hline 2013 & 1532 & 3.4 & 26.67 \\
\hline
\end{tabular}

Table 2: The frequencies of prostate cancer among males during: 2009-2013.

\begin{tabular}{|l|l|l|l|}
\hline Year & Frequency & $\begin{array}{l}\text { Within Total } \\
\text { Cancers (\%) }\end{array}$ & $\begin{array}{l}\text { Within Male } \\
\text { Cancers (\%) }\end{array}$ \\
\hline 2009 & 404 & 9 & 13.12 \\
\hline 2010 & 526 & 1.2 & 12.53 \\
\hline 2011 & 547 & 1.2 & 13.40 \\
\hline 2012 & 778 & 1.7 & 16.03 \\
\hline 2013 & 744 & 1.6 & 15.62 \\
\hline
\end{tabular}

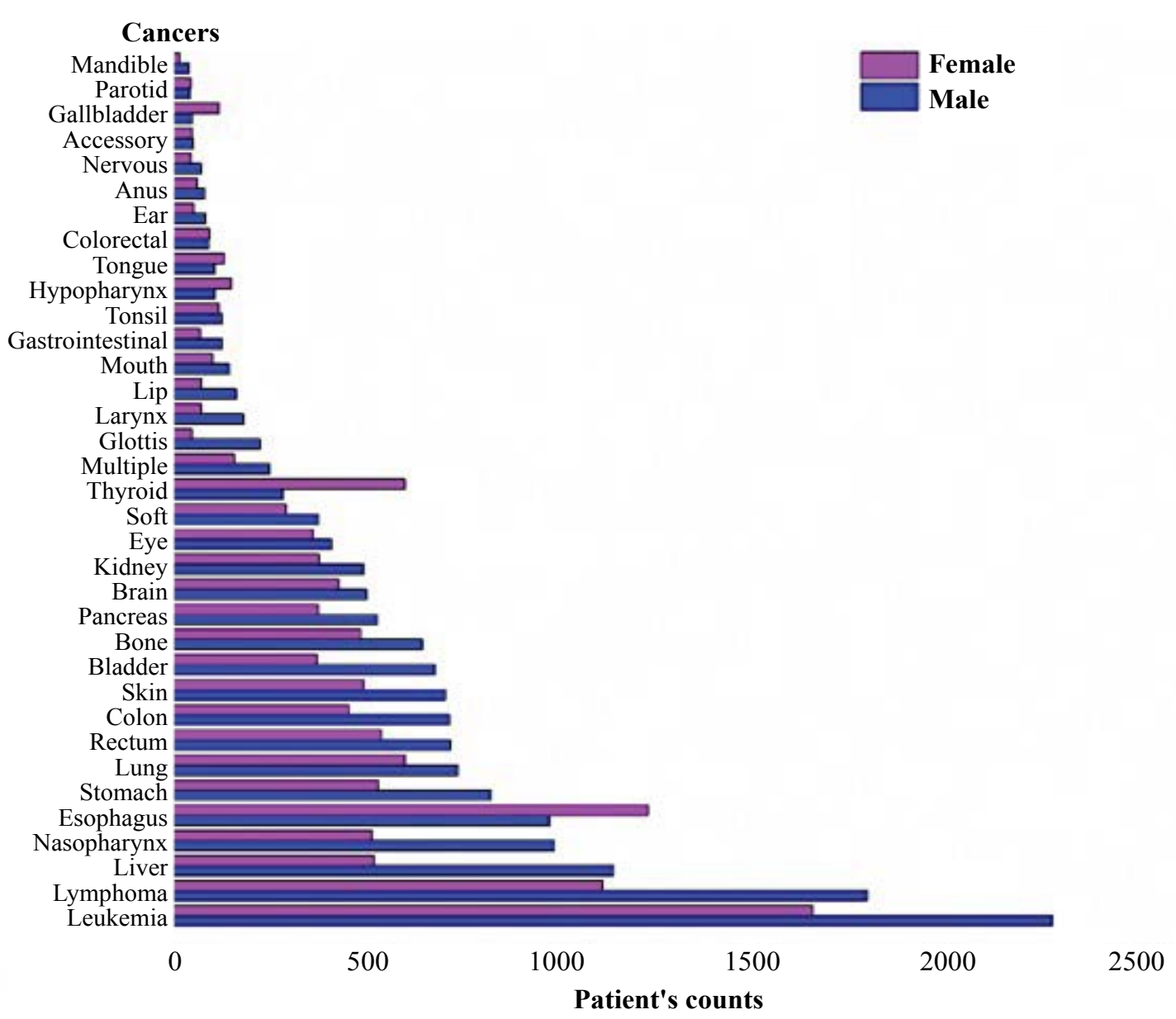

Figure 3: The most incident cancers in Sudan among males and females for the period 2009-2013. 
Male to Female Ratios for the major 35 cancers in Sudan during 2009-2013

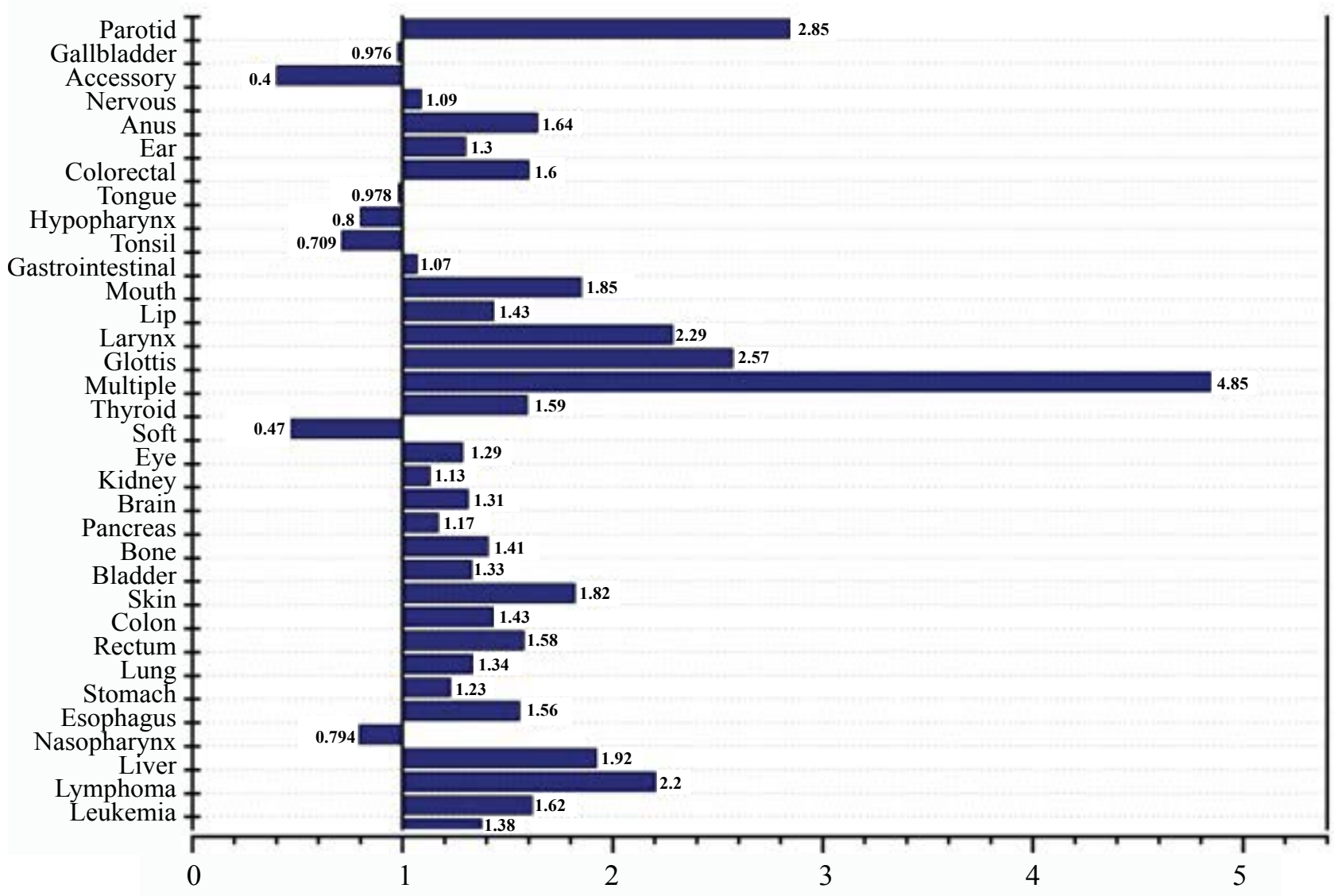

Figure 4: M: F ratios for the most common primary cancer sites by gender in Sudan from 2009 to 2013 (Sex specific cancers are excluded).

\section{Discussion}

The cancer susceptibility differences between women and men has long been ignored in determining cancer risk. However, recently it has been shown worldwide that men are more susceptible to cancer than women especially in hematological malignances and this disparity was attributed to many factors such as hormone and metabolism differences between women and men (REF). Accordingly, we investigated the disparities of gender regarding cancer incidences in Sudan. In our investigation, we used a population-based data that covers the entire Sudan over the period of five years (2009-2013). We found that during this period, a total of 45413 cancer cases (including gender-specific cancers) were recorded. Among these 20974 (46\%) cases were in males and 24439 (54\%) cases were in females. These results suggest that Sudanese males have a lower incidence of all cancers combined compared to females. Similar findings were reported previously in Ethiopia in which 540 malignant cancer cases were examined for gender differences. Of these $346(64 \%)$ cases were identified to be in females and 194 (36\%) cases were in males [12]. Likewise, more cancer cases were reported in female (370.1) compare to male (256.3) in Sub Saharan African countries (http://globocan.iarc.fr/Pages/ fact_sheets_population.aspx).
However, when we excluded the sex-specific cancers, the incidence of cancer cases of all other sitescombined were higher in men compared to women (Figure 4) suggesting that the increase number of cancer casesin female is due to the high number of breast cancer cases. In fact, breast cancer is the second most common cancer among women in Sub-Saharan Africa accounting for 16.8 percent of all female cancers, however, recently it surpassed cervical cancer as first most common cancer in many Sub-Saharan African countries $[13,14]$. Possible explanations for the higher proportion of breast cancer occurring in women include, differences in incidence pattern of cancers that occur commonly in both sexes compared to the age structure of the population; as well as the relative ease of diagnosis of common female cancers compared with those in men [15]. In addition, women have a more frequent contact with the health care system due to uptake of maternal/child health care services and a better medical care-seeking behavior compared to men [16].

In general cancer incidences among males are higher compared to those in females excluding sex-specific cancers, which was reported before according to the 2002 estimates of cancer incidence for the Sub-Saharan Africa region were the overall world-standardized cancer rates were 133 per 100000 females and 136 per 
100000 males [14]. Similarly, many studies revealed that hematologic malignancies are generally more common in males and that this can be generalized to most other cancers [6]. An analysis of the National Cancer Institute, Surveillance Epidemiology and End Results (SEER) database showed that incidence rates per 100000 people and age-adjusted to the 2000 US standard population for all cancers in the United States for 2004-2008 were 553.0 for males, and 416.5 for females. Yielding an incidence rate ratio (IRR) of 1.33 for all cancers [17] and 1.35 without sex specific cancers. Likewise, the lifetime probability of developing cancer is $44.85 \%$ for males, and $38.08 \%$ for females [6].

Contradictory results were found in Denmark where women ranked high for most types of cancer due to the fact that more than $40 \%$ of the labor force have been women since the 1970s and a large proportion of Danish women may have had occupational exposures, including exposures to carcinogens [18]. Similarly, cancer incidence data from two population-based cancer registries in Nigeria (the Ibadan Population-Based Cancer Registry and the Abuja Population-Based Cancer Registry) suggested that there was no significant difference in the distribution of cancers by sex between the 2 cancer registries (Fisher's exact test $p$-value $=0.89$ ). Both registries reported similar proportion of cases in males and in females [15].

Sex differences are also seen in other diseases such as diverticular, cardiovascular and diabetic diseases. Among patients with diverticular disease, sex differences have also been noted by Weizman and Nguyen [19]. The data showed that although diverticular disease is more common among men 50 years or younger, the incidence among women predominates in older age groups [20-22]. Sex differences in hypertension prevalence and cardiovascular risk factors of 352 healthy hospital workers in Nigeria reported thirty-five percent of participants were hypertensive, Men had a higher prevalence of hypertension (38.4\% vs. $33.0 \%$ ) and prehypertension ( $37.6 \%$ vs. $29.7 \%$ ). Gender bias is also an attribute of autoimmune diseases. Approximately 6.7 million American women are affected by autoimmune diseases compared to 1.8 million men. Population with the highest incidence of Type I diabetes Mellitus all show male excess. In contrast, Type II diabetes showed a female excess in the first half of the last century but seems to be equally prevalent among males and females today [23].

In summary, our study revealed that, among 45413 cancer cases of population-based data that cover the Sudan over the five-year period (2009-2013), 46\% were in male and $54 \%$ were in female. Including breast and prostate cancer, females appeared to have the highest number of incidences compared to males ( $\mathrm{M}: \mathrm{F}>$ 1). However, males exceeded females in the number of affected people in 28 out of 35 of non-specific sex can- cers. Therefore, the study illustrates that; males are significantly more susceptible to cancers in $80 \%$ of the top 35 cancers when excluding sex-specific cancers.

\section{Funding}

Sudan Atomic Energy Commission (SAEC) funded this work as part of human resources development programs.

\section{Acknowledgement}

I acknowledge the officers of Federal Ministry of Health, Sudan for their help in data collection.

\section{Conflict of Interest}

None.

\section{References}

1. Hamad HMA (2006) Cancer initiatives in Sudan. Ann Oncol 17: 32-36.

2. Forsea AM (2016) Cancer registries in Europe-going forward is the only option. Ecancermedicalscience 10: 1-15.

3. Mohammed ME, Hassan AM, Abdelhadi HA, Elsadig MG, Adam DM, et al. (2014) Burden and Pattern of Cancer in the Sudan, 2000-2006. Br J Med Med Res 4: 1231-1243.

4. Edgren G, Liang L, Adami HO, Chang ET (2012) Enigmatic sex disparities in cancer incidence. Eur $\mathrm{J}$ Epidemiol 27: 187-196.

5. Scelo G, Li P, Chanudet E, Muller DC (2017) Variability of Sex Disparities in Cancer Incidence over 30 Years: The Striking Case of Kidney Cancer. Eur Urol Focus.

6. Dorak MT, Karpuzoglu E (2012) Gender differences in cancer susceptibility: An inadequately addressed issue. Front Genet 3: 1-11.

7. Kautzky-Willer A, Harreiter J, Pacini G (2016) Sex and Gender Differences in Risk, Pathophysiology and Complications of Type 2 Diabetes Mellitus. Endocr Rev 37: 278316.

8. Cartwright RA, Gurney KA, Moorman AV (2002) Sex ratios and the risks of haematological malignancies. $\mathrm{Br} \mathrm{J}$ Haematol 118: 1071-1077.

9. Pearce MS, Parker L (2001) Childhood cancer registrations in the developing world: Still more boys than girls. Int J Cancer 91: 402-406.

10. Cook MB, McGlynn KA, Devesa SS, Freedman ND, Anderson WF (2011) Sex disparities in cancer mortality and survival. Cancer Epidemiol Biomarkers Prev 20: 1629-1637.

11. Zahm SH, Fraumeni Jr JF (1995) Racial, ethnic, and gender variations in cancer risk: Considerations for future epidemiologic research. Env Heal Perspect 103: 283-286.

12. Tefera B, Assefa M, Abebe B, Rauch D (2016) Patterns of Cancer in University of Gondar Hospital: North-West Ethiopia. J Oncol Med Pract 1: 2-5.

13. Sitas F, Parkin M, Chirenje Z, Stein L, Mqoqi N, et al. (2006) Disease and Mortality in Sub-Saharan Africa. ( $2^{\text {nd }}$ edn), World Bank, Washington DC, USA.

14. Vorobiof BDA, Sitas F, Vorobiof G (2001) Breast Cancer Incidence in South Africa. J Clin Oncol 19: 125-127.

15. Jedy-Agba E, Curado MP, Ogunbiyi O, Oga E, Fabowale T, et al. (2012) Cancer incidence in Nigeria: A report from 
population-based cancer registries. Cancer Epidemiol 36: 271-278.

16. Mills EJ, Bakanda C, Birungi J, Chan K, Hogg RS, et al. (2011) Male gender predicts mortality in a large cohort of patients receiving antiretroviral therapy in Uganda. J Int AIDS Soc 14: 1-7.

17. Siegel R, Naishadham D, Jemal A (2012) Cancer Statistics, 2012. CA Cancer J Clin 62: 10-29.

18. Rix BA, Skov T, Lynge E (1997) Socioeconomic group, occupation and incidence of breast cancer and genital cancer among women in Denmark. Eur J Public Health 7: 177-181.

19. Weizman AV, Nguyen GC (2011) Diverticular disease: Epidemiology and management. Can J Gastroenterol 25: 385-389.
20. Warner E, Crighton EJ, Moineddin R, Mamdani M, Upshur $R$ (2007) Fourteen-year study of hospital admissions for diverticular disease in Ontario. Can J Gastroenterol 21: 97 99.

21. Kang JY, Hoare J, Tinto A, Subramanian S, Ellis C, et al. (2003) Diverticular disease of the colon--on the rise: A study of hospital admissions in England between 1989/1990 and 1999/2000. Aliment Pharmacol Ther 17: 1189-1195.

22. Schauer PR, Ramos R, Ghiatas AA, Sirinek KR (1992) Virulent diverticular disease in young obese men. Am J Surg 164: 443-446.

23. Gale EAM, Gillespie KM (2001) Diabetes and gender. Diabetologia 44: 3-15. 